\title{
Design impact of acceptability and dependability in assisted living robotic applications
}

\author{
Filippo Cavallo ${ }^{1}$ (D) Raffaele Limosani $^{1} \cdot$ Laura Fiorini $^{1} \cdot$ Raffaele Esposito $^{1} \cdot$ Rocco Furferi $^{2} \cdot$ Lapo Governi $^{2}$. \\ Monica Carfagni ${ }^{2}$
}

Received: 14 February 2018 / Accepted: 5 March 2018

(c) Springer-Verlag France SAS, part of Springer Nature 2018

\begin{abstract}
This paper presents the implementation and investigation of a novel user centred method, adopted to design, develop and test a personal robot system, composed of a mobile robotic platform and a smart environment, for assisting people at home. As robots need to work closely with humans, novel interactive engineering design approaches are required to develop service robots that are adherent to end users' needs and that can be quickly employed in daily life. Particularly, this paper presents a methodology based on the simultaneous evaluation of dependability and acceptability, thus leading to an innovative approach for metrics and benchmarks that includes not only the main technical attributes of dependability, but also the parameters of acceptability, both implemented via a user-centered design and co-creative approach. Additionally, dependability and acceptability form the basis for defining standardized methodologies to test and evaluate robotic systems in dedicated experimental infrastructures (or robotic facilities), which are conceived to facilitate engineers in their studies and assessments.
\end{abstract}

Keywords Companion robot $\cdot$ Service robotics $\cdot$ User centred design $\cdot$ Dependability $\cdot$ Acceptability

\section{Introduction}

The market projections foresee a remarkable growth of service robotics for personal applications [1], particularly considering the current demographic changes and the need

$\triangle$ Filippo Cavallo

filippo.cavallo@santannapisa.it

Raffaele Limosani

raffaele.limosani@santannapisa.it

Laura Fiorini

laura.fiorini@santannapisa.it

Raffaele Esposito

raffaele.esposito@santannapisa.it

Rocco Furferi

rocco.furferi@unifi.it

Lapo Governi

lapo.governi@unifi.it

Monica Carfagni

monica.carfagni@unifi.it

1 The BioRobotics Institute, Scuola Superiore Sant'Anna, Pisa, Italy

2 Department of Industrial Engineering, University of Florence, Florence, Italy for innovative and sustainable social and health care services at home for individuals. In the not too distant future, robots will actively collaborate with humans in specific daily tasks such as, for example, management of daily activities [2], promotion of social inclusion [3], and suggestion of healthy activities [4]. As robots need to work closely with humans, novel interactive engineering design approaches are required to develop service robots that are adherent to end users' needs and can be quickly employed in daily life (e.g., taking inspiration from the service design thinking [5], which promotes the user-centered and co-creative design). Today, social robotics is a well-established branch of robotics. It involves the study of how to design, develop, and evaluate robots able to interact and cooperate with humans by embodying human social behaviors and rules and having high levels of acceptability in providing useful and efficient services [6]. In literature, several works focus on the analysis and evaluation of service robotics for personal applications [7,8]. Acceptability and dependability are fundamental parameters of the robot design process because they influence the reliability and success of the deployment of personal robots in real life. In this context, the aim of this paper is to present the implementation and investigation of a comprehensive user centred method adopted to design, develop, and evaluate a personal robotic 
system, which included both acceptability and dependability aspects during all the design phases. The final results of this research demonstrate the crucial importance to consider both of these factors and to evaluate them in a dedicated infrastructure for replicable experiments and objectively measurable metrics and benchmarks.

\section{Related works}

In literature, the investigation of acceptability and dependability aspects of service robotics for personal applications represents a crucial aspect for deployability. Research results have been published on varied associated topics including key theories on the acceptability of technologies, socioemotional requirements for acceptability, and evaluation of dependability and usability criteria in a realistic context.

Aspects concerning the acceptability of robots for personal assistance have been investigated beginning with the first studies of robotics applied to no-industrial use (e.g., MovAiD Project $[9,10])$. Important theories on the acceptability of technologies, such as the Technology Acceptance Models (TAM) [11] and the Unified Theory of Acceptance and Use of Technology (UTAUT) [12], were applied to the robotics context to identify which aesthetical and functional factors could influence the reliability of service robots and favor or inhibit the interaction and use of robots by end users. For example, Heerink et al. [13] analyzed and elaborated the UTAUT concepts to develop a toolkit based on twelve constructs (anxiety, attitude, facilitating conditions, intention to use, perceived adaptability, perceived enjoyment, perceived ease of use, perceived sociability, perceived usefulness, social influence, social presence and trust) that are useful to measure the acceptance of assistive robots by groups of users such as elderly people. Another interesting study investigated the socio-emotional requirements necessary in a shopping robot to be acceptable by users [14]. This research highlighted that extraversion, a human trait that is typically important in the shopping sector, is also a key feature to be considered in the design of this type of service robot because if favors interaction and feelings of safety for customers. Furthermore, in one study, Matarić investigated the factors for human-robot interaction (HRI) that are required to foster the acceptability of robotic platforms used to motivate older people during physical exercise [15]. The two experiments conducted in this study showed a strong user preference for the relational robot in terms of enjoyability, companionship, and motivational capabilities. Moreover, the European project Robot-Era, which was aimed at developing three service robots to support elderly people in their everyday lives, based the complete design process of the robotic platform on the acceptability and usability criteria and assessed them with older persons in a realistic context [16]. These experi- ments obtained positive feedback from end users in terms of robot acceptance and provided useful suggestions to improve the three robotic platforms and the tested services.

All these studies are only a part of the huge number of research projects conducted to date to investigate acceptability aspects in personal robotics. However, they are not sufficient to guarantee the successful deployment of robots in real life. Indeed, the development of personal robots must consider safety issues for users, the environment, and the robot itself [17]. For this reason, dependability is another important factor that should be pursued in robotic development.

Service robots that work in actual environments have a higher level of demands and requirements placed upon them compared to industrial robots. Foremost, service robotics requires that dependable robot systems must operate in human-inhabited environments and must accomplish their tasks with adequate performance and robustness in dynamic and unpredictable environments. In studies investigating the general dependability issues, human factors and human errors are recognized as important but are considered separately from technical issues of dependability. Particularly relevant is the safety requirement, because the robot actively and dynamically engages the environment and hence takes full responsibility in the case of hard contact with people or objects [18]. Adaptability, which is another parameter proposed by Sommerville et al., is the process of changing a system to configure it for its environment of use [19]. In contrast with the industrial context, robots working in the domestic and urban environments and with different users should be able to vary and correct their behavior in real time according to the changing conditions. Moreover, another important parameter to be considered in the service robotic field, data security, is a composite of confidentiality, the absence of unauthorized disclosure of information; and integrity, the absence of improper system state alterations [20].

While, for acceptability studies, much literature and various methods and models have been presented, to the best of our knowledge, few works have been presented addressing dependability for personal and domestic robotic applications. Furthermore, few works have addressed both acceptability and dependability and tried to investigate a correlation between them.

Bischoff et al. proposed in their analysis that the most important rule to consider is that a robot system exists for the purpose of providing value to its users. Therefore, before defining the specifics of any system, or determining the hardware platform or development processes, a question has to be answered: Does a planned feature contribute to the system's ability to provide value to the user? [21]. Scientists have become aware that one of the most important factors that will determine the acceptance of new technologies by the average users (and particularly elderly people) is to ensure that they are not pressured to take on new systems in which they 
Fig. 1 Scheme of the user-centered design and co-creative approach adopted during the entire process. Elderly volunteers were involved for the needs analysis in phase 1 and for evaluation of the system in phase 4

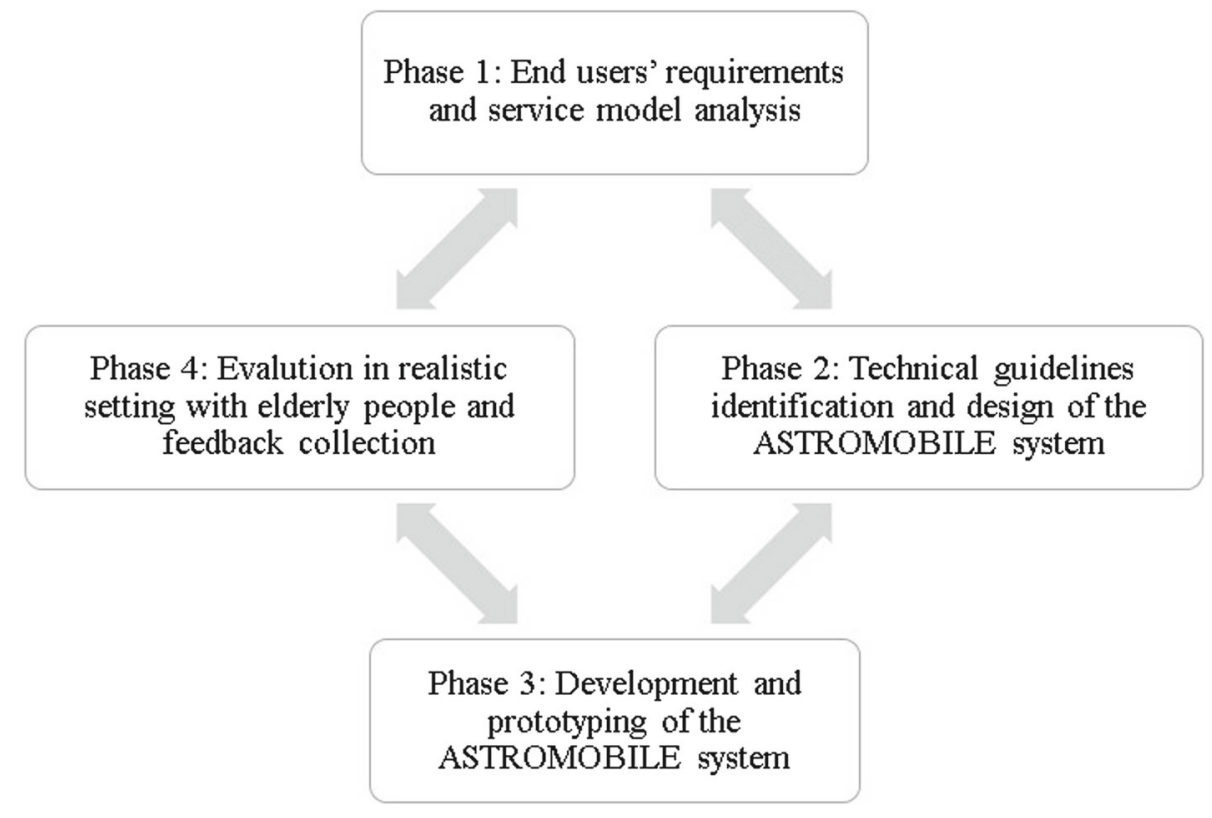

\section{Methods and instruments}

have no interest. This means that an environment of Service Robotics can be successful only if the potential users are willing to adopt it [22]. A robotic system that is unusable in a particular context by a particular user or which does not improve the overall quality of life for a user cannot and should not be considered to be dependable [19]. The technologies must be able to manage numerous challenges before they become practical and commercially viable in real life. Such challenges include certain functionality and usability issues, security and privacy concerns, architectural difficulties, and cost-benefit balancing.

In this context, this paper aims to demonstrate the importance of integrating technically focused dependability and human-oriented acceptability evaluations for deploying service robotics in real assisted environments. This leads to an innovative methodology for metrics and benchmarks that includes not only the main technical attributes of dependability, but also the parameters of acceptability, both implemented by using a user-centered design and a co-creative approach. With this purpose, this paper presents the process of designing, developing, and testing a home-based system, composed of a mobile robotic platform and a smart environment, for assisting elderly persons. First, a dependability model [23] was achieved to confirm technical specifications; second, an acceptability model was used to evaluate the human-oriented features [24]. Additionally, such a dependability and acceptability duo forms the basis for defining standardized methodologies to test and evaluate robotic systems in dedicated experimental infrastructures (or robotic facilities), which are conceived to facilitate roboticists in their studies and assessments.
This paper focuses on the design, development, and evaluation of the ASTROMOBILE system [25], a system conceived for favorable independent living, improved quality of life, and efficiency of care for senior citizens in domestic environments.

The development of the ASTROMOBILE system and its relative services were performed using a user-centered design and co-creative approach that have been demonstrated to be crucial for plausible deployment and exploitation in real socio-medical contexts [13]. In particular, the project was conducted using the four steps described in Fig. 1 [26].

With the use of ad-hoc structured questionnaires and focus-group sessions with elderly people, the functions of the system and the favored interfaces for the use and appearance (shape human-like, not human-like, dimensions, colors, materials) of the ASTRO robot to be perceived safe, friendly, and acceptable by senior citizens, were consolidated.

Concerning functions, elderly individuals deemed that the most useful robotic services are bringing and transporting objects, helping them to stand up from chairs and sofa, working as a calendar to remind them of appointments and times to take medicines, consulting with doctors or caregivers and home surveillance for security reason.

For the question, "How do you prefer to interact with the robot?" most elderly people chose the vocal interface and the remote controller because, for them, speaking is the most natural way to communicate. They would like the robot to be able to understand their vocal commands and also to reply with vocal messages. Furthermore, concerning the remote 

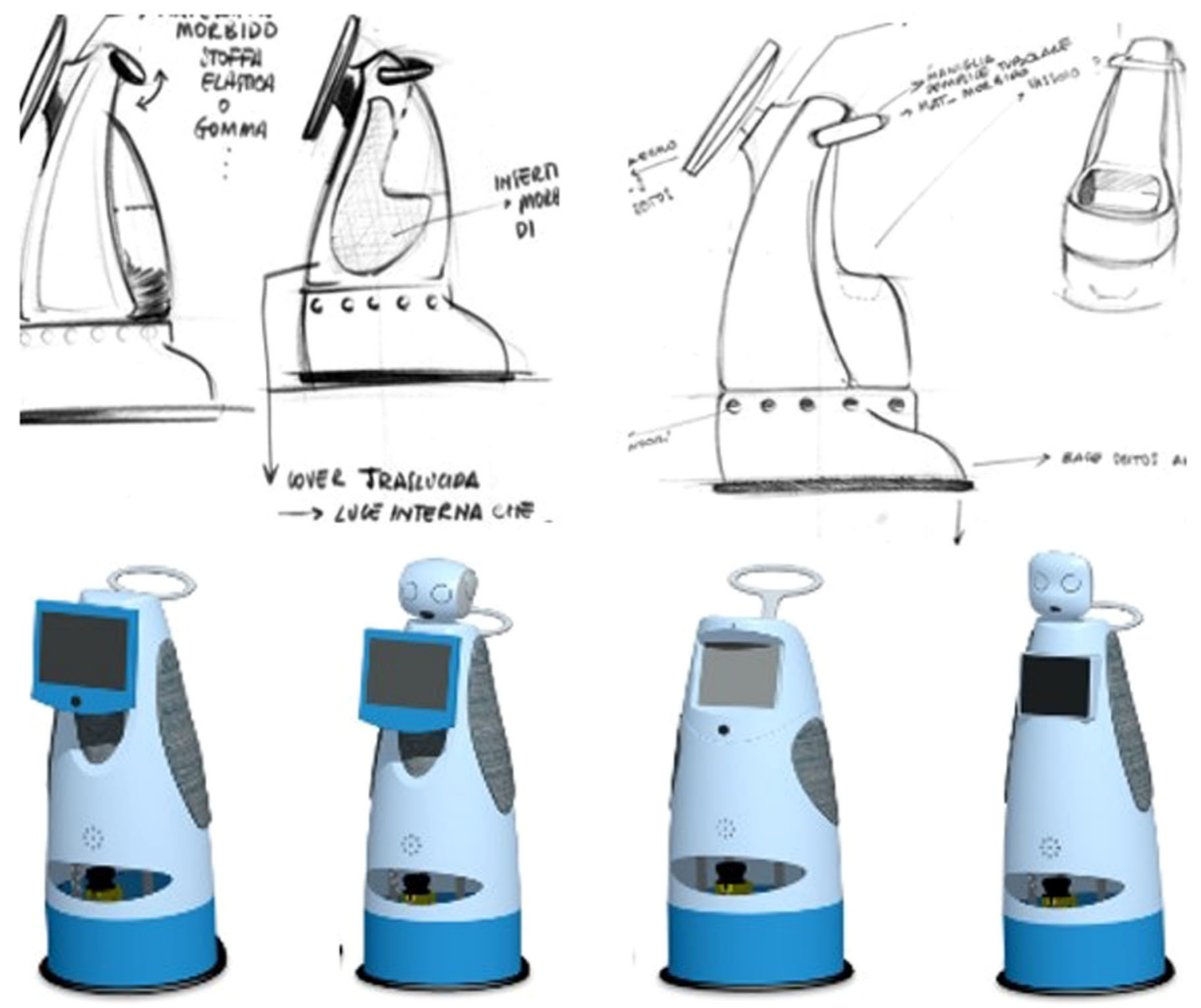

Fig. 2 The initial concept of the ASTRO robot in different versions

controller, most seniors are familiar with using a TV remote controller, so they suggested the use of a similar tool to control the robot.

During the focus groups with elderly individuals, four possible versions of the ASTRO robot (Fig. 2) were sketched to reach a consensus on the final version of the robot.

Most of the people commented that if the robot has characteristics similar to humans, it is perceived as friendlier. Regarding the size of the robot, elderly persons stated that they preferred medium dimensions (maximum height $1.50 \mathrm{~m}$ ) so they could watch the robot monitor easily without experiencing neck and cervical stress. Concerning the colors of the robot, the most popular colors chosen were blue and gray (Fig. 3). For the cover materials, the elderly subjects touched various types of materials (plastic, rubber, metal) having different consistencies (soft, rigid) and textures (smooth, rough). Then, the subjects were asked to choose the material for the cover. Most of the individuals preferred the combination of rigid material and spongy areas.

\subsection{System overview}

The ASTROMOBILE system was composed of two main coexisting agents that cooperated together and with end users: a mobile robotic platform, called ASTRO, and an Ambient Intelligent (AmI) infrastructure, implemented via wireless sensor networks for environmental monitoring and user localization. The ASTRO robot was built on the commercial mobile platform SCITOS G5 (Metralabs, Germany), which, based on a differential drive and a weight and payload of 60 and $50 \mathrm{~kg}$, respectively, was able to move at a rate of up to $1.1 \mathrm{~m} / \mathrm{s}$. ASTRO was equipped with a laser range sensor SICK S300 and a 9-axis inertial measurement unit (IMU) that allowed it to safely navigate in the domestic environment, avoiding obstacles and planning trajectories. The aesthetics, accessories, and interfaces were appropriately designed on the basis of the end users' requirements analysis, preliminarily identified in the first development phase. As a matter of fact, the ASTRO robot (Fig. 3) was designed with a human-like shape and stylized eyes and mouth. It was 
Fig. 3 ASTRO, the mobile robotic platform developed on the basis of acceptability and dependability requirements

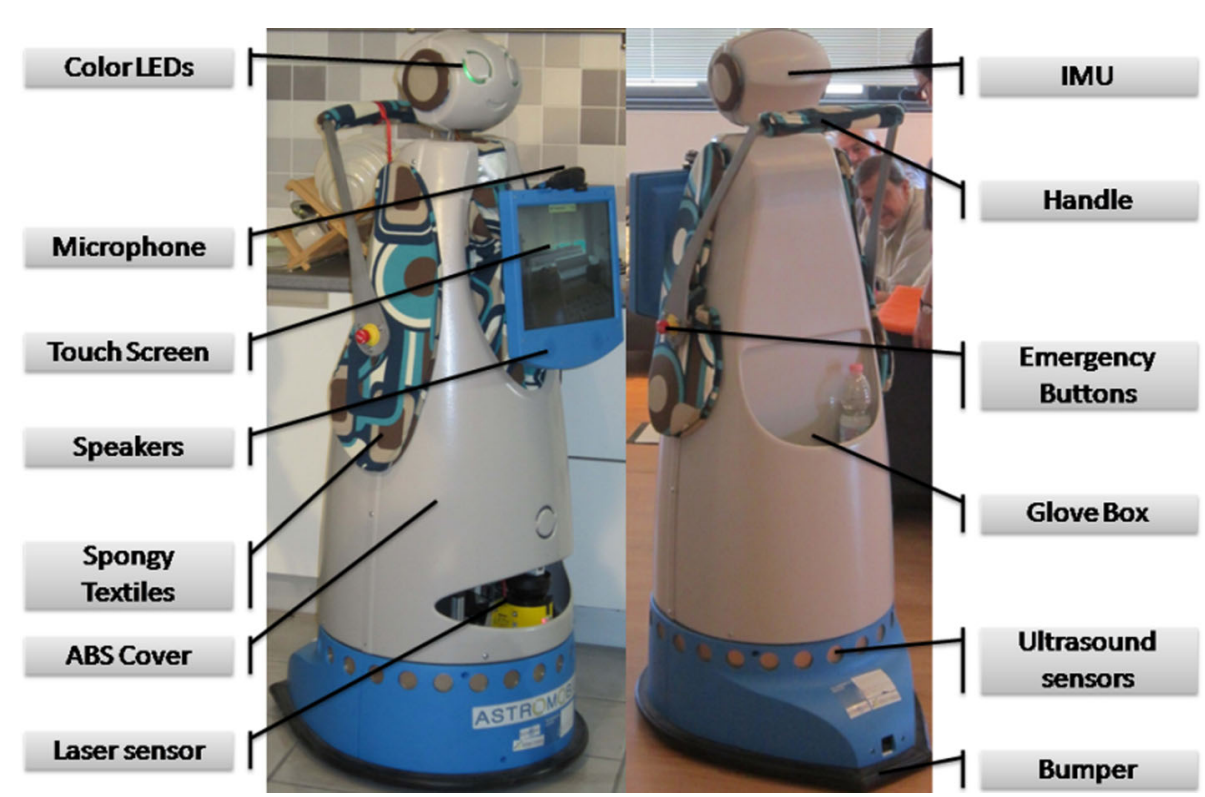

made of a gray rigid thermoplastic ABS material and some lateral interchangeable spongy textiles on the chest and head. To favor the interaction with end users, ASTRO included an adjustable touch screen, red/blue/green-colored LEDs inside the eyes, and a flexible microphone and a set of speakers for the speech recognition.

The AmI infrastructure was implemented via two wireless sensor networks, suited to monitor the environment (sensor network, SN) and localize the users (localization network, LN). SN and LN were designed as two different modular mesh networks on the ZigBee channels 26 and 20 (Ember ZigBee-Pro stack), respectively, to improve the robustness and reliability of the networks.

The localization cycle $(5 \mathrm{~Hz})$ consisted of the measurement of the received signal strength indication (RSSI) from all anchor nodes (ANs) of the LN, in occasion of the reception of a message from the mobile node $(\mathrm{MN})$, and next in the transmission of such RSSIs to the data logger (DL), where the position of the MN was computed. SN monitored the environment by means of appropriate sensor nodes on doors, beds, and sofas. Particularly, the information from these sensors was used to identify the presence of end users on beds or sofas, and this information was then combined with data from $\mathrm{LN}$

\subsection{Experimental setting}

Fifteen elderly people ( 3 male and 12 female), between 66 and 84 years of age $(74.87 \pm 6.48)$, were involved in the experimentation. Seven participants had a low educational level and eight had a high level of education; eight of them were married and seven were widowed. All participants completed the test session and the questionnaire.
Table 1 The standard actions behind a service

\begin{tabular}{|c|c|}
\hline Actors & Actions \\
\hline User, ASTRO & $\begin{array}{l}\text { The User is somewhere in the home; ASTRO is } \\
\text { in the standby position in the home }\end{array}$ \\
\hline AmI & $\begin{array}{l}\text { The AmI reveals an event and triggers ASTRO to } \\
\text { start the service for the User }\end{array}$ \\
\hline AmI & $\begin{array}{l}\text { The AmI calculates the User's position by means } \\
\text { of the two sensor networks and sends it to } \\
\text { ASTRO }\end{array}$ \\
\hline ASTRO & $\begin{array}{l}\text { ASTRO calculates the path to reach the User and } \\
\text { navigate in the home, avoiding any obstacles }\end{array}$ \\
\hline User, ASTRO & $\begin{array}{l}\text { When ASTRO reaches the User, the service is } \\
\text { provided }\end{array}$ \\
\hline
\end{tabular}

The work was performed in the permanent infrastructure of the DomoCasa Lab, which reproduces a fully furnished apartment of approximately $120 \mathrm{~m}^{2}$ and represents an appropriate workplace to test and measure benchmarks, allowing replication and comparison for robot performance.

Two scenarios were considered for the experimental setting. In the first scenario, the user calls ASTRO because he/she needs it to carry out some daily activities (i.e., stand up from the sofa, call a friend, etc.). In the second scenario, ASTRO autonomously helps the user as consequence of a particular event (i.e., remind them to take a medicine, notify of an alert in the home, etc.). In both cases, the scenarios are composed of the actions presented in Table 1.

\subsection{Dependability evaluation}

Dependability is defined as the property or the ability of a system to deliver a service that can justifiably be trusted, to avoid 
failures that are more frequent or more severe and outage durations that are longer than is acceptable to the users [5]. A systematic exposition of the concepts of dependability consists of three parts: impairments affecting dependability, means for pursuing dependable systems, and attributes that comprise dependability [27]. The term "dependability" is here conceived with such attributes as reliability, availability, safety, adaptability, maintainability, and security. Depending on the specific application context, more emphasis has to be placed on each of the attributes included in the concept of dependability.

In this work, the dependability model was defined by identifying appropriate metrics and benchmarks that describe some technical means that are the basis of the implementation of the standard actions described in Table 1. The technical requirements for dependability of the social robot are:

- Navigation capability to move from a starting point to the target user with maximum efficiency (as quickly as possible), avoid any obstacle (without delaying the service delivery time), move gently (perceived safe by the user).

- Accuracy in measuring the user's position in the home environment so the robot can safely and effectively interact with the user.

\subsection{Acceptability evaluation}

Acceptability is defined as "the demonstrable willingness within a user group to employ technology for the tasks it is designed to support" [28]. The most used model for the acceptability evaluation is the Unified Theory of Acceptance

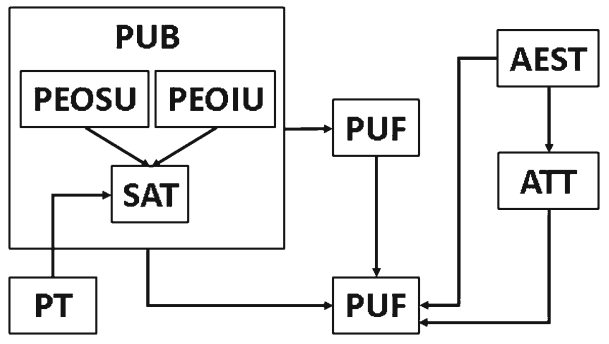

Fig. 4 Overview of the model used to evaluate the acceptability

Use of Technology (UTAUT) [29]. The UTAUT aims to explain user intentions to employ a technological system and subsequent usage as the result of the interaction of several key constructs. However, UTAUT was developed for technology acceptance evaluation at work, so the questionnaire should be adapted to a robot usage in a domestic environment by adapting, omitting, or adding questions [30]. Among the different techniques used to evaluate the acceptability, we used the evaluation kit proposed by [13]. To assess the proposed system acceptance, a proper model was designed and applied (Fig. 4) in which three main factors, perceived usability (PUB), perceived usefulness (PUF), and attitude (ATT), influenced the intention to use (ITU). The validation of the model was based on analysis of the data collected during the experiment to relate the data to scores showing the indicated intention to use the proposed system.

Starting from the UTAUT model and the results achieved in [13], an ad-hoc questionnaire for the ASTROMOBILE system was developed to investigate the constructs included in our model. The constructs and the interrelations between them, assumed as hypothesis, are shown in Table 2 and Fig. 4,
Table 2 Constructs used to build the acceptability model

\begin{tabular}{|c|c|c|}
\hline Code & Construct & Definition \\
\hline PUB & Perceived usability & $\begin{array}{l}\text { Usability is the perceived quality of a user's } \\
\text { experience when interacting with the system }\end{array}$ \\
\hline PEOIU & Perceived ease of interface use & $\begin{array}{l}\text { The degree to which the user believes that using } \\
\text { the interface system would be free of effort }\end{array}$ \\
\hline PEOSU & Perceived ease of service use & $\begin{array}{l}\text { The degree to which the user believes that using } \\
\text { the services would be free of effort }\end{array}$ \\
\hline SAT & Satisfaction & $\begin{array}{l}\text { Pleasure or contentment derived from such system } \\
\text { usage }\end{array}$ \\
\hline PT & Perceived time & $\begin{array}{l}\text { Perceived time spent by the robot to perform the } \\
\text { service }\end{array}$ \\
\hline PUF & Perceived usefulness & $\begin{array}{l}\text { The degree to which a person believes that using } \\
\text { the system would enhance his or her daily } \\
\text { activities }\end{array}$ \\
\hline ATT & Attitude & $\begin{array}{l}\text { Positive or negative feelings about using the } \\
\text { technology }\end{array}$ \\
\hline AEST & Aesthetics & $\begin{array}{l}\text { Positive or negative feelings about the aesthetics } \\
\text { of the robot }\end{array}$ \\
\hline ITU & Intention to use & $\begin{array}{l}\text { The intention to use the system at the present or in } \\
\text { the future }\end{array}$ \\
\hline
\end{tabular}


Table 3 The main parameters for dependability evaluation

\begin{tabular}{|c|c|c|c|c|c|c|}
\hline & \multicolumn{2}{|c|}{ Kitchen } & \multicolumn{2}{|c|}{ Kitchen with obstacle } & \multicolumn{2}{|c|}{ Bedroom } \\
\hline & Mean & SD & Mean & SD & Mean & SD \\
\hline $\mathrm{T}_{\mathrm{s}}(\mathrm{s})$ & 18.833 & 0.723 & 20.460 & 0.404 & 29.40 & 0.346 \\
\hline PL [m] & 9.601 & 0.046 & 10.260 & 0.169 & 14.76 & 0.118 \\
\hline $\mathrm{V}_{\mathrm{m}}(\mathrm{m} / \mathrm{s})$ & 0.510 & 0.064 & 0.501 & 0.418 & 0.502 & 0.341 \\
\hline $\mathrm{S}_{\mathrm{p}}(\%)$ & 98.988 & 0.110 & 93.511 & 1.393 & 88.42 & 0.636 \\
\hline $\mathrm{J}_{\mathrm{p}} / 10^{6}$ & 0.023 & 0.011 & 0.013 & 0.002 & 0.02 & 0.003 \\
\hline
\end{tabular}

respectively. Furthermore, statements about gender, age, education, and marital status were added to enable investigation of their influence on the other constructs of the model. Each user involved in the experiment replied to the statements on a 5-point Likert type scale, in which a participant's high rate of agreement corresponded to 4 or 5 points and a low one to 1 or 2 points, while 3 points indicated that the user did not form an opinion.

The processing of questionnaire data included the following steps:

- The Cronbach's alpha was calculated to understand the reliability of the construct. An alpha of at least 0.7 is considered acceptable [31].

- Basic descriptive statistics were employed to initially assess the statement results.

- The Pearson test was applied to verify the hypothesis presented in the model.

- The Mann-Whitney $U$-test was performed to compare different conditions or users.

\section{Dependability measurements}

\subsection{Social navigation parameters}

The presence of humans in the robots' workspace requires novel approaches that consider the constraints of human comfort as well as social rules. In the literature, several navigation methods have been proposed [32], analyzing social conventions (i.e., proxemics), human-like behaviors for robots that are used to define design and development guidelines for socially acceptable robots. The aforementioned methods are based on the assessment of how motion is perceived regarding different conditions such as speed, distance, and orientation. Starting from this approach, three possible parameters were identified to test the robot performance:

- Time to delivery service $\left(\mathrm{T}_{s}\right)$ : the time it takes the robot from the trigger of the service from the planner to initial provision of the service. Mainly, it is the time for the robot to move from the starting point to the user's position.
- Straightness of path $\left(\mathrm{S}_{p}\right)$ : the ratio of the straight line connecting the starting point of the robot in its workspace (position ' 0 ') and the target point (user position), and the actual path length (PL) (total distance traveled to reach the target). In the case of complex paths, where the starting and target points are not in sight, then a broken line is considered.

- Mean velocity $\left(\mathrm{V}_{m}\right)$ : the ratio between PL and $\mathrm{T}_{s}$.

- Jerk of the path $\left(\mathrm{J}_{p}\right)$ : a measure of the motion smoothness; it is a rate of change in acceleration. It is normalized for different task durations and path lengths:

$$
J_{s}=\sqrt{\frac{T^{5}}{2 * D^{2}} \int_{0}^{T}\left[\frac{d^{3} x^{2}}{d t^{3}}+\frac{d^{3} y^{2}}{d t^{3}}\right] d t}
$$

Five experimental trials were performed with the robot moving from the starting position to each of the selected end positions in the DomoCasa Lab. The first position was selected in the kitchen and was reached twice, with and without an obstacle along the path. The second position was selected in the bedroom. Table 3 depicts the measured parameters for the three different trials (Fig. 5a).

\subsection{User localization}

The user localization provides fundamental information so robots can promptly deliver the service inside the working place. Over the past decades, the indoor localization technologies based on wireless sensor networks (WSNs) have gradually increased their importance in different aspects of people's daily lives [33], including assisted living, navigation, emergency detection, surveillance, shopping, and many other location-based services (LBS). The accuracy of measuring the user position is one of the most important parameters used to evaluate the performance of a robot in delivering a service. Particularly with service robotics, it is not necessary to have millimeter scale accuracy, but an appropriate standard must be achieved to favor the interaction of human and robot during the provision of the service. For example, in [34] it was stated that the user positions should be provided with at least room-level localization resolution 
Fig. 5 a The path covered by the robot to test the navigation capabilities. b The circular pre-planned path users were asked to follow, starting from and finishing at the sofa, passing through the kitchen (twice), bathroom, and bedroom. The localization accuracy was measured at the fixed points, highlighted by the small circles (a)

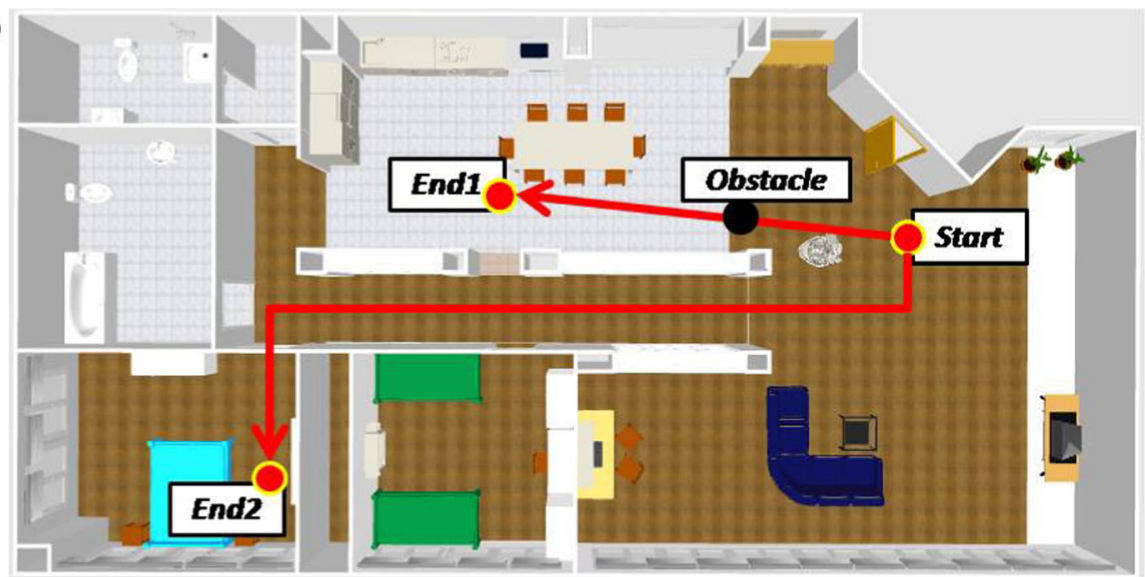

(b)

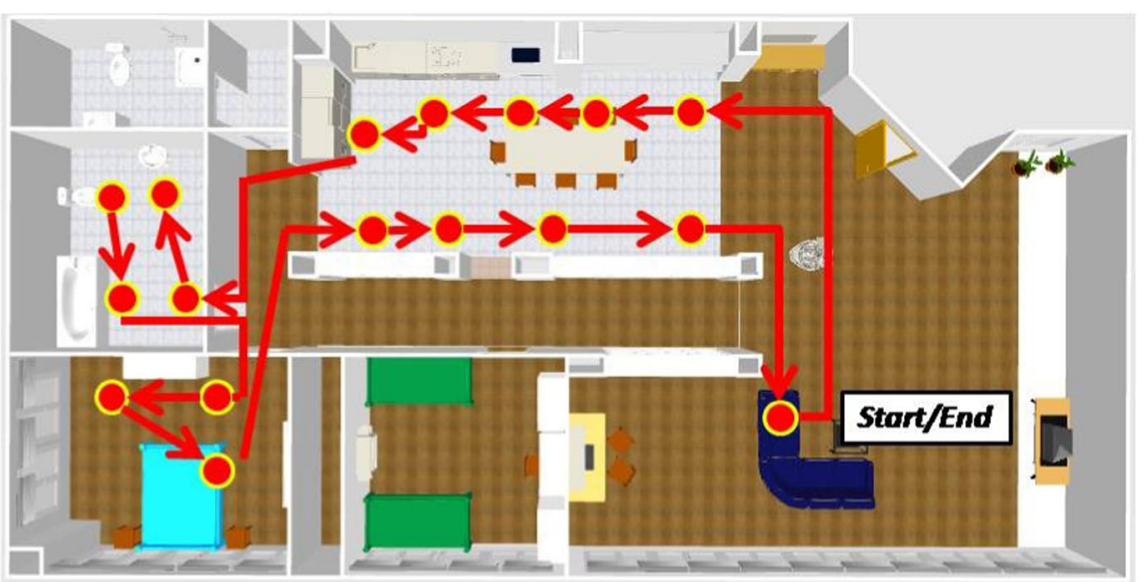

(granularity), while in the EVAAL (Evaluating AAL Systems through competitive benchmarking) competition [35], the localization accuracy was positively evaluated only if lower than $4 \mathrm{~m}$. Because the ASTROMOBILE system uses a Kinect motion-sensing input device for human robot interaction, and considering that the optimal distance between robot and user consists of between 1.2 and $3.5 \mathrm{~m}$ [36], it was defined to reach a localization accuracy of $2 \mathrm{~m}$ so the robot would be working in the middle of the optimal Kinect depth range. Starting from these considerations, one possible parameter was identified to test the robot performance:

- Localization accuracy (LA): the accuracy was measured as the difference between the actual and the estimated user position.

The user localization module (ULM) provided a multi-user localization service to collect and fuse data from the LN and SN. A sensor fusion approach based on a Kalman filter [37] for user localization was implemented by exploiting both range-free [38] and range-based [39] localization methods, according to [16]. Presence sensors were used to improve positioning accuracy and perform host detection. Numeric values $(\mathrm{x}, \mathrm{y})$ and semantic information on user position were provided to the robots as a service. To investigate the accuracy of the localization service, users wore an $\mathrm{MN}$ and moved over a pre-planned trajectory, from the living room to the bedroom and back within an overall localization workspace of $92 \mathrm{~m}^{2}$. Start and end points of the trajectory coincided; the user crossed the kitchen and the bathroom standing on 18 specific positions of interest, marked as dots in Fig. 5b.

Seven experimental trials were performed to provide a consistent dataset and highlight the advantages of the proposed sensor fusion approach and system architecture. The mean localization error was $0.98 \mathrm{~m}$, and the root mean square error (RMSE) was $1.22 \mathrm{~m}$.

\section{Acceptability analysis}

The evaluation of the main constructs, defined in the model of Fig. 4, were positive with an average value always higher than 3.4 (Table 4). The perceived usefulness (PUF) achieved the best result, demonstrating that the services provided by the robot could positively influence the users' lives. Both the perceived ease of interface use (PEOIU) and perceived ease of service use (PEOSU) are positive, leading to a good perceived usability (PUB). Slightly lower is the attitude to 
Table 4 Cronbach's alpha and descriptive statistics

\begin{tabular}{llllll}
\hline Construct & Alpha Cronbach & MIN & MAX & MEAN & SD \\
\hline ATT & 0.920 & 2 & 5 & 3.8000 & 0.9579 \\
PUF & 0.426 & 1 & 5 & 4.3667 & 1.0128 \\
PUB & 0.826 & 1 & 5 & 3.9267 & 1.1179 \\
PEOIU & 0.855 & 1 & 5 & 3.9334 & 1.2505 \\
PEOSU & 0.891 & 1 & 5 & 3.9238 & 1.0624 \\
SAT & 0.957 & 2 & 5 & 3.7334 & 0.9433 \\
ITU & 0.646 & 1 & 5 & 3.4000 & 1.3797 \\
\hline
\end{tabular}

Table 5 Correlational scores

\begin{tabular}{llll}
$\begin{array}{l}\text { Independent } \\
\text { variables }\end{array}$ & $\begin{array}{l}\text { Dependent } \\
\text { variables }\end{array}$ & $\begin{array}{l}\text { Pearson } \\
\text { correlation }\end{array}$ & $\begin{array}{l}\text { Sig. (2-tailed) } \\
<0.05\end{array}$ \\
\hline ATT & ITU & 0.304 & 0.270 \\
PUF & & 0.506 & 0.054 \\
PUB & & 0.510 & 0.052 \\
PUB & PUF & 0.135 & 0.631 \\
PEOIU & SAT & 0.489 & 0.064 \\
PEOSU & & 0.633 & 0.011 \\
\hline
\end{tabular}

use the services (ATT is 3.8) and even lower is the intention of use, which achieved the lowest value (ITU is 3.4).

To verify the constructs' reliability, the Cronbach's alpha was calculated. Table 4 shows that five constructs out of seven were reliable because they have alpha values of at least 0.7 . Nevertheless, alpha over 0.6 can be considered acceptable, as shown in [40], so the ITU construct could be considered, as well.

To evaluate the hypothesis of the model presented in Fig. 4, the Pearson test was used to show all the correlations between constructs (Table 5). By analyzing the correlations with the right significativity, it is possible to highlight that the SAT derives more from PEOSU than from PEOIU. Alternatively, even at the limits, PUF and PUB imply ITU.

Considering external factors such as gender (male or female), age (under or over age 75), education (low or high level), and marital status (married or not) and applying the Mann-Whitney $U$-test, we found a significant difference between the two conditions that influenced all constructs except for the marital status, as shown in Table 6. Furthermore, a positive or negative judgment on aesthetics of the robot influenced the score of ITU and ATT, and the user's satisfaction was significantly related their perception about time spent by the robot to perform the service.

\section{Discussion and conclusions}

This paper presents a comprehensive analysis of dependability and acceptability of a personal robotic system integrated in smart environments to assist the aging population at home. To the best of our best knowledge, no literature has been published addressing both issues and their relationships. Dependability and acceptability are two crucial properties that should be considered for a successful design and consequent adoption of personal service robotics. This has been proved in the past separately with works focusing on or exclusively covering the analysis of dependability, claiming safety and robustness of robotic systems, or exclusively on the analysis of acceptability, promoting human-oriented interaction, social requirements, and graceful appearance as the basis of their acceptance. For acceptability, the Unified Theory of Acceptance Use of Technology (UTAUT), which is a consolidated standard $[28,29]$ and that has extensively been investigated in the field of service robotics by Heenrink [13], was used as starting point of this study. For dependability, instead, enlightenment was taken from those works [17-24] that defined appropriate metrics, aiming at measuring social navigation capabilities of robots working close to humans, robustness of systems to engage with users and delivery time of services. Additionally, the ISO/DIS 18646-1 and the ISO 13482 standards, respectively related to requirements and guidelines for the inherently safe design, protective measures, and information for use of personal care robots and the locomotion performance of wheeled robots in indoor environments, were used as reference to conduct dependability measurements.

In our opinion, these two aspects are intimately related, and their relationship is at the basis of a user-centered and cocreative design approach in the personal robotics sector [5]. In our approach, a multi-stakeholder working group [41],

Table 6 Mann-Whitney $U$-test (Sig. $<0.05$ )

\begin{tabular}{lllllll}
\hline & Gender & Age & Education & Marital status & Aesthetics & Perceived time \\
\hline ATT & $1.47 \times 10^{-06}$ & $2.23 \times 10^{-06}$ & $2.14 \times 10^{-06}$ & 0.767 & $1.1 \times 10^{-06}$ & - \\
PUF & $1.53 \times 10^{-06}$ & $2.32 \times 10^{-06}$ & $2.23 \times 10^{-06}$ & 0.768 & - & - \\
PUB & $1.53 \times 10^{-06}$ & $2.33 \times 10^{-06}$ & $2.23 \times 10^{-06}$ & 0.768 & - & - \\
ITU & $2.48 \times 10^{-04}$ & $1.50 \times 10^{-04}$ & $9.65 \times 10^{-05}$ & 0.375 & $-37 \times 10^{-06}$ & - \\
SAT & - & - & - & - & & - \\
\hline
\end{tabular}


composed of end users, care professionals, technical developers, and producers, has contributed in the main phases of the development and evaluation.

The high rate obtained in most of the constructs highlights that the general acceptability of the system from subjects is positive (Table 4). The co-creative and usercentered approach, used from the beginning of the design phase to identify functional specifications and appearance of the robot [42], was at the basis of the positive perception of the usefulness of the system. However, a bit lower acceptability performance was identified in terms of usability of the system, which led to a lower rate in ITU. For example, a moderate positive relationship is seen between PUF/PUB and ITU, confirming that the usefulness and usability of the system could influence the intention to use the system. This moderate result could be better explained in terms of construct by the fact that a lower correlation is found between the use of interfaces (PEOIU) and satisfaction (SAT) with respect to the usefulness of services (PEOSU). Concretely, subjects found difficulties in using the system and, from their comments, we noted that it was not easy to push small buttons on the graphical interfaces, the quality and volume of speakers of the robot were not easily understandable (sometime they asked to raise the volume), and the robot was slow in navigation.

This latter point, concerning the low speed of the robot, could be better analyzed considering the dependability metrics in Table 3. The maximum speed of the robot, set via software, was $0.6 \mathrm{~m} / \mathrm{s}$. This value was defined on the basis of experience gained by Knight et al. [43], which demonstrated that a robot navigating in fast conditions in office environments with a speed of $0.75 \mathrm{~m} / \mathrm{s}$ was better accepted and used than the same robot navigating in slow conditions with $0.3 \mathrm{~m} / \mathrm{s}$ speed. In our work, we decided to set the maximum speed of the robot a bit lower because of the stricter spaces of the home environment and the presence of older subjects for safety issues. As a matter of fact, Table 3 shows that the mean speed of the robot during the three experimental scenarios was approximately $0.5 \mathrm{~m} / \mathrm{s}$ due to the presence of obstacles or irregular paths. This value of speed was considered unacceptable, because the time to reach the user after the call was perceived as too long. To improve the SAT, it is necessary to explore other navigation approaches that permit the robot to move with higher speed while maintaining the safety of users.

On the other hand, the smoothness of navigation along the home paths was fairly good, with high values of Sp and low values of Jp. This is confirmed also by the acceptability point of view, because for the question asking if the robot was perceived to be safe during the testing phase, the users answered that the robot appeared friendly and innocuous.

Socio-demographic factors have a significant impact on the evaluation of the acceptance of personal care robots from elderly people, but experience with technology could mitigate their effects on acceptance [44]. In our case, elderly subjects were involved in the users' needs analysis, in the definition of functionalities and characteristics of the system, and in the testing phase (i.e., involved in almost all the co-creative approaches). This enabled elderly subjects with a low technological familiarity, particularly with robots, to more easily interact with the robot, thus leading to a general positive acceptability.

These results, however, could be affected by some restrictions due to the limited number of subjects involved and the short time the subjects interacted with the robot during the experimental phase. In particular, the significativity of the acceptability results could suffer in terms of Cronbach's alpha for validation, and the short-time experiments could have given an inaccurate perception of the robot working conditions. Nevertheless, these results give a promising, even if preliminary, confirmation that a comprehensive methodology to design and develop personal care robots, including dependability and acceptability, is an essential element of the user-centered and co-creative design approach. In future studies, a longer-term experiment and a larger group of test subjects will be arranged.

Finally, it should be noted that, from the point of view of engineers, the proposed evaluation framework is based on the possibility to have an experimental infrastructure that allows permanently maintaining and updating a number of integrated robotic platforms and pervasive sensors in the environment. Indeed, this framework exploits a system architecture composed of different layers, each of them provided by a software interface that enables engineers or scientists to collect experimental data and implement appropriate evaluation models and algorithms for benchmarks. The importance of a permanent infrastructure revolves also around the possibility to attract any stakeholder or customer, proving realistic scenarios to measure benchmarks. This facilitates the experimental procedures, including possible training phases for users.

\section{Appendix}

The following questions were used for each basic construct of the acceptability model, with answers ranked according to the Likert scale from 0 to 5 :

ITU

Q1 Do you think ASTRO robot can integrate itself with your habits and your lifestyle?

Q2 Would you buy ASTRO robot, now or in the future? 


\section{PEOIU}

Q1 Was the Touchscreen Interface easy to use?

Q2 Was the Microphone Interface easy to use?

Q3 Was the Smartphone Interface easy to use?

\section{PEOSU}

Q1 Was the Support in standing up service easy to perform?

Q2 Was the Object transport service easy to perform?

Q3 Was the Communication service easy to perform?

Q4 Was the Entertainment service easy to perform?

Q5 Was the Reminder service easy to perform?

Q6 Was the Environmental alert service easy to perform?

Q7 Was the Remote control for caregiver service easy to perform?

\section{PUF}

Q1 Was the Support in standing up service useful?

Q2 Was the Object transport service useful?

Q3 Was the Communication service useful?

Q4 Was the Entertainment service useful?

Q5 Was the Reminder service useful?

Q6 Was the Environmental alert service useful?

Q7 Was the Remote control for caregiver service useful?

Q8 Do you think a service robot, such as ASTRO, is useful?

\section{SAT}

Are you satisfied in:

Q1 Support in standing up service?

Q2 Object transport service?

Q3 Communication service?

Q4 Entertainment service?

Q5 Reminder service?

Q6 Environmental alert service?

Q7 Remote control for caregiver service?

\section{References}

1. Service Robotics Market by Operating Environment (Aerial, Ground, Marine) -2022| MarketsandMarkets (2017). Retrieved March 5, 2018, from https://www.marketsandmarkets.com/ Market-Reports/service-robotics-market-681.html

2. Fiorini, L., Esposito, R., Bonaccorsi, M., Petrazzuolo, C., Saponara, F., Giannantonio, R., De Petris, G., Dario, P., Cavallo, F.: Enabling personalised medical support for chronic disease management through a hybrid robot-cloud approach. Auton. Robots 41, 1263-1276 (2017). https://doi.org/10.1007/s10514-016-9586-9

3. Reppou, S., Karagiannis, G.: Social inclusion with robots: a RAPP case study using NAO for technology illiterate elderly at Ormylia Foundation, pp. 233-241. Springer, Cham (2015). https://doi.org/ 10.1007/978-3-319-15847-1_23
4. Fasola, J., Mataric, M.: A socially assistive robot exercise coach for the elderly. J. Hum. Robot Interact. 2(2), 3-32 (2013). https:// doi.org/10.5898/JHRI.2.2.Fasola

5. Stickdorn, M., Schneider, J. (Economist).: This is service design thinking: basics, tools, cases. BIS Publishers (2011). Retrieved March 5 2018, from http://www.bispublishers.com/thisis-service-design-thinking- $2 . \mathrm{html}$

6. García-Soler, Á., Facal, D., Díaz-Orueta, U., Pigini, L., Blasi, L., Qiu, R.: Inclusion of service robots in the daily lives of frail older users: a step-by-step definition procedure on users' requirements. Arch. Gerontol. Geriatr. 74, 191-196 (2018). https://doi.org/10. 1016/j.archger.2017.10.024

7. Robinson, H., MacDonald, B., Broadbent, E.: The role of healthcare robots for older people at home: a review. Int. J. Soc. Robot. 6, 575-591 (2014)

8. Dessimoz, J.-D., Gauthey, P.-F.: Domestic service robots in the real world: more on the case of intelligent robots following humans. Commun. Comput. Inf. Sci. 161, 88-101 (2011)

9. Dario, P., Guglielmelli, E., Laschi, C., Teti, G.: Technology and disability. Technology and Disability, vol. 10. Andover Medical Publishers (1991). Retrieved March 12 2018, from https://content. iospress.com/articles/technology-and-disability/tad00009

10. Arras, K.O., Cerqui, D.: Do we want to share our lives and bodies with robots? A 2000 people survey. Technical Report 605-1 (2005). https://doi.org/10.3929/ETHZ-A-010113633

11. Davis, F.D.: Perceived usefulness, perceived ease of use, and user acceptance of information technology. MIS Q. 13(3), 319 (1989). https://doi.org/10.2307/249008

12. Venkatesh, V., Morris, M.G., Davis, G.B., Davis, F.D.: User acceptance of information technology: toward a unified view. MIS Q. 27, 425-478 (2003)

13. Heerink, M., Krose, B., Evers, V., Wielinga, B.: Measuring acceptance of an assistive social robot: a suggested toolkit. In: RO-MAN 2009-The 18th IEEE International Symposium on Robot and Human Interactive Communication, pp. 528-533. IEEE (2009). https://doi.org/10.1109/ROMAN.2009.5326320. http:// ieeexplore.ieee.org/document/5326320/

14. Ludewig, Y., Doring, N., Exner, N.: Design and evaluation of the personality trait extraversion of a shopping robot. In: 2012 IEEE RO-MAN: The 21st IEEE International Symposium on Robot and Human Interactive Communication, pp. 372-379. IEEE (2012). https://doi.org/10.1109/ROMAN.2012. 6343781. http://ieeexplore.ieee.org/document/6343781/

15. Fasola, J., Matarić, M.J.: Using socially assistive human-robot interaction to motivate physical exercise for older adults. Proc. IEEE 100(8), 2512-2526 (2012)

16. Cavallo, F., Limosani, R., Manzi, A., Bonaccorsi, M., Esposito, R., Di Rocco, M., Pecora, F., Teti, G., Saffiotti, A., Dario, P.: Development of a socially believable multi-robot solution from town to home. Cogn. Comput. 6(4), 954-967 (2014)

17. Guiochet, J., Machin, M., Waeselynck, H.: Safety-critical advanced robots: a survey. Robot. Auton. Syst. 94, 43-52 (2017)

18. Caselli, S., Monica, F., Reggiani, M.: YARA: A software framework enhancing service robot dependability. In: Proceedings of the 2005 IEEE International Conference on Robotics and Automation, pp. 1970-1976. IEEE (2005). https://doi.org/10.1109/ROBOT. 2005.1570402

19. Sommerville, I., Dewsbury, G.: Dependable domestic systems design: a socio-technical approach. Interact. Comput. 19(4), 438 456 (2007). https://doi.org/10.1016/j.intcom.2007.05.002

20. Zamojski, W., Mazurkiewicz, J., Sugier, J., Walkowiak, T., Kacprzyk, J.: Complex Systems and Dependability, vol. 170. Springer, Berlin (2012). https://doi.org/10.1007/9783-642-306624

21. Bischoff, R., Graefe, V.: Design principles for dependable robotic assistants. Int. J. Humanoid Rob. 1(1), 95-125 (2004) 
22. Metsis, V., Le, Z., Lei, Y., Makedon, F.: Towards an evaluation framework for assistive environments. In: Proceedings of the 1st ACM International Conference on PErvasive Technologies Related to Assistive Environments-PETRA '08, 1 (2008). https://doi.org/ 10.1145/1389586.1389601

23. Boussaada, Z., Curea, O., Camblong, H., Bellaaj Mrabet, N., Hacala, A.: Multi-agent systems for the dependability and safety of microgrids. Int. J. Interact. Des. Manuf. 10(1), 1-13 (2016)

24. Matsas, E., Vosniakos, G.-C.: Design of a virtual reality training system for human-robot collaboration in manufacturing tasks. Int. J. Interact. Des. Manuf. 11(2), 139-153 (2017)

25. Cavallo, F., Aquilano, M., Bonaccorsi, M., Mannari, I., Carrozza, M.C., Dario, P.: Multidisciplinary approach for developing a new robotic system for domiciliary assistance to elderly people. In: Proceedings of the Annual International Conference of the IEEE Engineering in Medicine and Biology Society, EMBS, Vol. 2011, pp. 5327-5330. IEEE (2011). https://doi.org/10.1109/ IEMBS.2011.6091318

26. Cavallo, F., Aquilano, M., Bonaccorsi, M., Limosani, R., Manzi, A., Carrozza, M.C., Dario, P.: Improving domiciliary robotic services by integrating the ASTRO robot in an AmI infrastructure. Springer Tracts Adv. Robot. 94, 267-282 (2014). https://doi.org/10.1007/ 978-3-319-02934-4_13

27. Avizienis, A., Laprie, J.-C., Randell, B., Landwehr, C.: Basic concepts and taxonomy of dependable and secure computing. IEEE Trans. Depend. Secure Comput. 1(1), 11-33 (2004)

28. Dillon, A.: Human acceptance of information technology. In: International Encyclopedia of Ergonomics and Human Factors, pp. 1105-1108. Taylor and Francis, London (2006). Retrieved from March 5 2018, http://arizona.openrepository.com/arizona/handle/ $10150 / 105880$

29. Venkatesh, V., Morris, M.G., Davis, G.B., Davis, F.D.: User acceptance of information technology: toward a unified view. MIS Q. 24, 425-478 (2003)

30. Heerink, M., Kröse, B.J.A., Evers, V., Wielinga, B.J.: Studying the acceptance of a robotic agent by elderly users. Int. Assist. Robot. Mechatron. 7(3), 33-43 (2006)

31. Santos, J.R.A.: Cronbach's alpha: a tool for assessing the reliability of scales. J. Extension 37(2), 1-5 (1999)

32. Lichtenthäler, C., Peters, A., Griffiths, S., Kirsch, A.: Social navigation-identifying robot navigation patterns in a path crossing scenario. In: Lecture Notes in Computer Science (including subseries Lecture Notes in Artificial Intelligence and Lecture Notes in Bioinformatics), vol. 8239 LNAI, pp. 84-93. Springer, Cham (2013). https://doi.org/10.1007/978-3-319-02675-6_9

33. Gu, Y., Lo, A., Niemegeers, I.: A survey of indoor positioning systems for wireless personal networks. IEEE Commun. Surv. Tutor. 11(1), 13-32 (2009). https://doi.org/10.1109/SURV.2009.090103
34. Eisa, S., Moreira, A.: Requirements and metrics for location and tracking for ambient assisted living. In: 2012 International Conference on Indoor Positioning and Indoor Navigation, IPIN 2012-Conference Proceedings, pp. 1-7. IEEE (2012). https://doi. org/10.1109/IPIN.2012.6418935

35. Potortì, F., Park, S., Jiménez Ruiz, A., Barsocchi, P., Girolami, M., Crivello, A., Macias-Guarasa, J.: Comparing the performance of indoor localization systems through the EvAAL framework. Sensors 17(12), 2327 (2017). https://doi.org/10.3390/s17102327

36. Livingston, M.A., Sebastian, J., Ai, Z., Decker, J.W.: Performance measurements for the Microsoft Kinect skeleton. In: 2012 IEEE Virtual Reality (VR), vol. 298(704), pp. 119-120 (2012). https:// doi.org/10.1109/VR.2012.6180911

37. Bonaccorsi, M., Fiorini, L., Cavallo, F., Saffiotti, A., Dario, P.: A cloud robotics solution to improve social assistive robots for active and healthy aging. Int. J. Soc. Robot. 8(3), 393-408 (2016)

38. Wang, Y., Jin, Q., Ma, J.: Integration of range-based and rangefree localization algorithms in wireless sensor networks for mobile clouds. In: Proceedings-2013 IEEE International Conference on Green Computing and Communications and IEEE Internet of Things and IEEE Cyber, Physical and Social Computing, GreenCom-iThings-CPSCom 2013, pp. 957-961. IEEE (2013). https://doi.org/10.1109/GreenCom-iThings-CPSCom.2013.165

39. Arias, J., et al.: Malguki: an RSSI based ad hoc location algorithm. Microprocess. Microsyst. 28.8, 403-409 (2004)

40. Hayes, A.F., Krippendorff, K.: Answering the call for a standard reliability measure for coding data. Commun. Methods Meas. 1(1), 77-89 (2007). https://doi.org/10.1080/19312450709336664

41. Briand, R., Fischer, X., Arrijuria, O., Terrasson, G.: Multidisciplinary design process based on virtual prototyping for microsystem design. Virtual Phys. Prototyp. 5(3), 153-162 (2010). https:// doi.org/10.1080/17452759.2010.504084

42. Dore, R., Pailhes, J., Fischer, X., Nadeau, J.P.: Identification of design variables and criterion variables towards the integration of user requirements into preliminary design. Int. J. Prod. Dev. 4(5), 508 (2007). https://doi.org/10.1504/IJPD.2007.013045

43. Knight, H., Veloso, M., Simmons, R.: Taking candy from a robot: Speed features and candy accessibility predict human response. In: Proceedings-IEEE International Workshop on Robot and Human Interactive Communication, vol. 2015-Novem, pp. 355-362. IEEE (2015). https://doi.org/10.1109/ROMAN.2015.7333606

44. Flandorfer, P.: Population ageing and socially assistive robots for elderly persons: the importance of sociodemographic factors for user acceptance. Int. J. Popul. Res. 2012, 1-13 (2012). https://doi. org/10.1155/2012/829835 